\title{
Productos frontera. Metodología de clasificación en inspección farmacéutica como garantía de salud pública y buen gobierno
}

\author{
Bernardo Prieto Muñoz ${ }^{1, *}$ y María del Carmen Vidal Miñana ${ }^{2}$ \\ 1 Funcionario de Carrera Cuerpo de Farmacéuticos Titulares, Jefe de Sección de Inspección Farmacéutica, \\ Doctorando en el Departamento de Farmacia y Tecnología Farmacéutica y Parasitología de la Universidad \\ de Valencia, Diplomado en Salud Pública por la Escuela Nacional de Sanidad-Instituto de Salud Carlos III; \\ ORCID id: https://orcid.org/0000-0002-7174-8695 \\ 2 Técnica de Inspección de Seguridad Alimentaria, Doctora en Farmacia por el Departamento de Medicina \\ Preventiva, Salud Pública, Bromatología, Toxicología y Medicina Legal de la Universidad de Valencia, \\ Diplomada en Salud Pública por la Escuela Nacional de Sanidad-Instituto de Salud Carlos III; ORCID id: \\ https://orcid.org/0000-0002-2960-935X \\ * Autor correspondencia: bjprietom@gmail.com
}

DOI: https://doi.org/10.37536/RIECS.2021.6.2.278

Recibido: 6/9/2021; Aceptado: 25/10/2021; Publicado: 30/11/2021

Resumen: La expresión producto frontera se refiere a un producto difícil de clasificar administrativamente, por compartir características de dos o más categorías simultáneamente (alimento, medicamento, producto sanitario, cosmético y biocida). El objetivo de este trabajo fue la elaboración de una metodología de clasificación de los productos frontera. Se trata de un estudio observacional descriptivo. La estrategia del análisis consistió en la revisión caso por caso en base de la definición completa de cada producto establecida en la legislación nacional y de la Unión Europea considerando los aspectos que diferencian a cada categoría. Los elementos clave diferenciadores fueron: a) el mecanismo de acción principal para productos sanitarios frontera con medicamentos; b) la finalidad y el mecanismo de acción para productos sanitarios, cosméticos, equipos de protección individual y juguetes; c) el lugar de acción para cosméticos frontera con alimentos; d) las características del producto y la acción principal y secundaria para cosméticos frontera con biocidas. Con este procedimiento se consiguió la detección de errores de clasificación o fraudes por la intención de obviar los trámites de autorizaciones más estrictas de los medicamentos respecto a alimentos, productos sanitarios y cosméticos cuya comercialización es más económica y sencilla constituyendo un problema prioritario de salud pública. Los medicamentos no son inocuos y pueden producir efectos perjudiciales de los que es necesario advertir.

Palabras Clave: Productos frontera, Productos sanitarios, Medicamentos, Inspección farmacéutica, Salud pública.

Abstract: The expression borderline products refer to a difficult product to classify administratively,
for sharing characteristics of two or more categories simultaneously (food, medicine, medical
device, cosmetic and biocide). The aim of this work is the preparation of a methodology of
Classification of the borderline products. This is a descriptive observational studio. The analysis'
strategy consisted on a case by case review based in the complete definition of each product set up
in the national and the European Union legislation taken account of the aspects that differentiate
each category. The keys differentiating elements were: a) the mechanism of main action for the
medical devices borderline with medicinal products; b) the intended purpose and the mode of
action for the medical devices, the cosmetics products and the personal protective equipment; c) the
place of action for cosmetics borderline with foods; d) the characteristics of the product and the
primary of secondary action for cosmetics borderline biocides. With this procedure achieved the
detection of errors of classification or frauds by the intention of avert the formalities of permissions 
stricter of the medicines with regard to foods, medical devices and cosmetics whose commercialization is more economic and simpler constituting an important problem of public health. The medicines are not innocuous and can produce hurtful effects of which is necessary to warn.

Key words: Borderline products, medical devices, Medicines, Pharmaceutical inspection, Public health.

\section{Introducción}

\subsection{Inspección farmacéutica en frontera de la Unión Europea (UE)}

La Inspección Farmacéutica en frontera de la Unión Europea (UE) realiza las funciones de control y vigilancia de los géneros medicinales con el fin de que no supongan ningún riesgo para la salud pública.

La autoridad sanitaria de referencia en nuestro país para ciudadanos y profesionales sanitarios en materia de garantías de calidad, seguridad, eficacia, información y accesibilidad de los medicamentos y los productos sanitarios a fin de garantizar la protección y promoción de la salud de las personas es la Agencia Española de Medicamentos y Productos Sanitarios (AEMPS) [1].

\subsection{Concepto de producto frontera}

Existen definiciones legales de medicamento, alimento, producto sanitario, cosmético o biocida. La expresión producto frontera, no indica una categoría concreta y legal, sino aquel producto que, por diferentes motivos, es difícil de clasificar por compartir características de varias categorías simultáneamente [2].

La primera vez que se mencionó el concepto de producto frontera en un texto legal europeo fue en la Directiva 2004/27/CE: “A fin de mantener un nivel elevado de exigencias de calidad, seguridad y eficacia de los medicamentos de uso humano. Para tener en cuenta, por una parte, la aparición de nuevas terapias y, por otra, el número creciente de productos denominados "frontera" entre el sector de los medicamentos y los demás sectores, resulta conveniente modificar la definición de medicamento para evitar dudas sobre la legislación aplicable cuando un producto responda plenamente a la definición de medicamento pero pudiera responder también a la definición de otros productos regulados".

La comercialización de medicamentos está más controlada que la de los productos sanitarios y cosméticos. Existe una regulación comunitaria de aplicación en toda la UE, que permite asegurar la calidad, seguridad y eficacia de los medicamentos autorizados.

Es importante tener presente el control de la publicidad (Ley 10/2013) que se hace de algunos de estos productos a los que se atribuyen propiedades de salud que no han sido probadas [3].

La AEMPS considera prioritario que en el ámbito de la UE se clasifiquen de igual modo los productos frontera por el impacto que ocasionarían sobre la salud pública las distintas interpretaciones. Estos casos se tratan y discuten dentro del Grupo de Expertos de Productos Sanitarios en Frontera y Clasificación y se publican [4,5] como Manual on borderline and classification in the community regulatory framework for medical devices que sin tener carácter legislativo es una guía de consulta actualizada periódicamente con todas las propuestas para armonizar la clasificación de los géneros medicinales. 


\subsection{Principios para la correcta clasificación de un producto frontera}

Tres son los principios que se deben seguir:

- Principio de no acumulación, un producto no puede pertenecer a dos categorías simultáneamente salvo excepciones en las que se aplican ambas regulaciones.

- Principio de inspección "caso por caso", la inspección debe realizarse producto a producto, teniendo en cuenta la totalidad de sus características.

- Principio de jerarquía, si un producto responde a dos definiciones se aplica la legislación que tenga prioridad. Si un producto puede ser medicamento será medicamento.

\section{Objetivos}

Los objetivos de este trabajo son:

- Elaborar una metodología de clasificación de los productos frontera para proceder a una correcta categorización de los mismos.

- Detección de errores de clasificación o fraudes por la intención de obviar los tramites de autorizaciones más estrictas de los medicamentos respecto a alimentos, productos sanitarios, cosméticos, biocidas u otros productos.

\section{Material y Métodos}

\subsection{Tipo de estudio}

Se trató de un estudio observacional descriptivo.

\section{2. Ámbito y sujetos: sistema informático SIFAEX y expedientes}

Los expedientes objeto de estudio fueron aquellos iniciados por los operadores económicos en el Sistema Informático de Inspección Farmacéutica de Sanidad Exterior (SIFAEX) de aquellos productos de uso humano de los que existían duda entre dos categorías y eran considerados inicialmente frontera.

\subsection{Variables: definiciones de las categorías de productos}

Las definiciones de las distintas categorías de productos según la normativa nacional y de la Unión Europea que se tienen en cuenta para la correcta clasificación de un producto son las siguientes:

\subsubsection{Medicamento}

Queda definido en el art. 1. 2 de la Directiva 2004/27/CE como:

a) Toda sustancia o combinación de sustancias que se presente como poseedora de propiedades para el tratamiento o prevención de enfermedades en seres humanos, o

b) Toda sustancia o combinación de sustancias que pueda usarse en, o administrarse a seres humanos con el fin de restaurar, corregir o modificar las funciones fisiológicas ejerciendo una acción farmacológica, inmunológica o metabólica, o de establecer un diagnóstico médico.

El concepto de medicamento tiene una gran importancia. El que un producto tenga la consideración jurídica de medicamento significa que su comercialización, distribución, venta y publicidad van a quedar sometidos a una inspección administrativa mucho más intensa que la prevista para otros productos. Si se trata de medicamentos, las restricciones a la libertad empresarial y a la libre circulación de mercaderías, por otro lado, las garantías de salud para los ciudadanos también son mayores. La dificultad de precisar cuándo se trata de un medicamento es una cuestión controvertida, sobre todo tras la aparición de los denominados "productos frontera", como es el caso 
de muchos alimentos manipulados genéticamente con la finalidad de dotarlos de propiedades beneficiosas para la salud.

Analizando la definición legal de medicamento, se utilizan dos criterios: el del modo que se presenta el producto y el de la función que éste puede cumplir.

\subsubsection{Medicamento por su presentación}

Este concepto puede causar sorpresa, se puede pensar que un producto debe ser lo que es y no como se presente, al menos jurídicamente. La finalidad del criterio de presentación como señala el Tribunal de Justicia de la Unión Europea en su sentencia STCE de 16 de abril de 1991 es "combatir el curanderismo". Este concepto "está destinado a abarcar no solo los medicamentos nocivos o tóxicos, sino también los diversos productos utilizados, en lugar de los remedios adecuados", pues el consumo de estos productos puede hacer que los pacientes se alejen de los medicamentos eficaces, defendiendo, de esta forma, tanto la salud como el bolsillo de los ciudadanos.

\subsubsection{Medicamento por su función}

Los productos que se consideren como medicamentos entrañan un riesgo para la salud humana superior a la de otros productos. Este riesgo justifica el sometimiento a controles rigurosísimos, como señala el TJCE en su histórica sentencia ya referida, los medicamentos son "sustancias que pueden tener incidencia sobre el funcionamiento del organismo y pueden tener consecuencias sobre la salud en general".

La Directiva 2004/27/CE (artículo 2) y el Real Decreto Legislativo 1/2015 (artículo 8.6) introdujeron un apartado clarificador:

“En caso de duda, cuando un producto pueda responder a la definición de medicamento se le aplicará esta ley, incluso si a dicho producto se le pudiera aplicar la definición contemplada en otra norma."

La calificación como medicamento o como otro producto debe hacerse "caso por caso", y no con carácter general. Los inspectores farmacéuticos deben examinar cada preparado en concreto, teniendo en cuenta la totalidad de sus características, y, en particular, su composición, sus propiedades farmacológicas, su modo de empleo, el conocimiento que de él tengan los consumidores $\mathrm{y}$, sobre todo, los riesgos que pueden provocar su uso.

\subsubsection{Alimento}

A efectos del Reglamento n⿳0 178/2002 se entenderá por alimento “cualquier sustancia o producto destinados a ser ingeridos por los seres humanos o con probabilidad razonable de serlo, tanto si han sido transformados entera o parcialmente como si no".

La definición de alimento no incluye ni a los medicamentos ni a los cosméticos (artículo 2, apartados a y e). Un producto, según el principio de exclusión, o es alimento o es medicamento.

\subsubsection{Producto sanitario}

Según el Reglamento (UE) 2017/745 se entiende por producto sanitario: “todo instrumento, dispositivo, equipo, programa informático, implante, reactivo, material u otro artículo destinado por el fabricante a ser utilizado en personas, por separado o en combinación, con alguno de los siguientes fines médicos específicos:

- Diagnóstico, prevención, seguimiento, predicción, pronóstico, tratamiento o alivio de una enfermedad,

- Diagnóstico, seguimiento, tratamiento, alivio o compensación de una lesión o de una discapacidad,

- Investigación, sustitución o modificación de la anatomía o de un proceso o estado fisiológico o patológico, 
- Obtención de información mediante el examen in vitro de muestras procedentes del cuerpo humano, incluyendo donaciones de órganos, sangre y tejidos

y que no ejerce su acción principal prevista en el interior o en la superficie del cuerpo humano por mecanismos farmacológicos, inmunológicos ni metabólicos, pero a cuya función puedan contribuir tales mecanismos".

Los siguientes productos también se considerarán productos sanitarios:

- Los productos de control o apoyo a la concepción,

- Los productos destinados específicamente a la limpieza, desinfección o esterilización de los productos sanitarios, los accesorios de productos sanitarios y los productos sin finalidad médica enumerados en el anexo XVI.

\subsubsection{Accesorio de un producto sanitario}

Según el Reglamento (UE) 2017/745 se define como:

“Un artículo que, sin ser en sí mismo un producto sanitario, está destinado por su fabricante a ser usado de forma conjunta con uno o varios de dichos productos, para permitir específicamente que el producto o productos sanitarios puedan utilizarse con arreglo a su finalidad prevista o para contribuir específica y directamente a la funcionalidad médica de los productos sanitarios a efectos de su finalidad prevista".

El presente Reglamento no será aplicable a los productos sanitarios para diagnóstico in vitro regulados por el Reglamento (UE) 2017/746, a los medicamentos, a los cosméticos y a los alimentos.

\subsubsection{Cosmético}

El Reglamento CE nº 1223/2009 define los productos cosméticos como: “toda sustancia o mezcla destinada a ser puesta en contacto con las partes superficiales del cuerpo humano (epidermis, sistema piloso y capilar, uñas, labios y órganos genitales externos) o con los dientes y las mucosas bucales, con el fin exclusivo o principal de limpiarlos, perfumarlos, modificar su aspecto, protegerlos, mantenerlos en buen estado o corregir los olores corporales."

El RD 209/2005 sobre productos cosméticos transcribe prácticamente la misma definición y considera que son productos cosméticos decorativos los que figuran en el anexo $\mathrm{V}$, "por poseer sustancias coloreadas y por su poder cubriente, se aplican sobre diferentes zonas del cuerpo para acentuar temporalmente su belleza o enmascarar o disimular diversas imperfecciones cutáneas".

\subsubsection{Productos de cuidado personal}

Estos productos no se encuentran armonizados en la UE. La Disposición adicional segunda del RD 209/2005 define los productos de cuidado personal “todas las sustancias o preparados que, sin tener la consideración legal de cosméticos, biocidas, productos sanitarios o medicamentos, están destinados a ser aplicados sobre la piel, dientes o mucosas del cuerpo humano con finalidad higiénica o estética o para neutralizar o eliminar ectoparásitos". En este grupo se incluyen productos tales como dentífricos, productos de estética, pediculicidas, hidratantes vaginales, limpiadores anales, productos para el masaje deportivo, limpiadores nasales u oculares, o cualquier otro producto que pueda ser calificado como tal".

\subsubsection{Biocidas de uso personal y clínico}

La Agencia Española de Medicamentos y Productos Sanitarios (AEMPS) publica periódicamente una lista de los biocidas autorizados. Por tanto, es difícil situarlos como producto frontera.

Según el Reglamento 528/2012 un biocida es:

- “Toda sustancia o mezcla, en la forma en que se suministra al usuario, que esté compuesto por, o genere, una o más sustancias activas, con la finalidad de destruir, contrarrestar o neutralizar 
cualquier organismo nocivo, o de impedir su acción o ejercer sobre él un efecto de control de otro tipo, por cualquier medio que no sea una mera acción física o mecánica,

- toda sustancia o mezcla generada a partir de sustancias o mezclas distinta de las contempladas en el primer guion, destinada a ser utilizada con la intención de destruir, contrarrestar o neutralizar cualquier organismo nocivo, o de impedir su acción o ejercer sobre él un efecto de control de otro tipo, por cualquier medio que no sea una mera acción física o mecánica.

Un artículo tratado que tenga una función biocida primaria se considerará un biocida".

\subsection{Fuentes de información: manual de productos frontera y clasificación $[4,5]$}

Es otra herramienta de consulta para la clasificación en caso de duda de un producto frontera.

\subsection{Estrategia de análisis}

Se analizó caso por caso todos los expedientes de los productos frontera presentados para su tramitación.

Se tuvieron en cuenta, en base a la legislación nacional y comunitaria de la UE que se relaciona en el anexo I, lo siguiente:

- La definición completa de cada producto incidiendo en aspectos clave diferenciadores tales como mecanismo de acción, modo de administración, lugar de aplicación y finalidad.

- Consideración de elementos legales adicionales (jerarquías).

- Consideraciones de exclusiones legales.

- Las características del producto: indicaciones, etiquetado, composición, normas de calidad y si existen varios componentes, se diferencia entre la acción principal y la acción coadyuvante y/o auxiliar.

- Otras herramientas de apoyo en caso de duda fueron las directrices de las autoridades sanitarias y la consulta de manuales de productos frontera. 


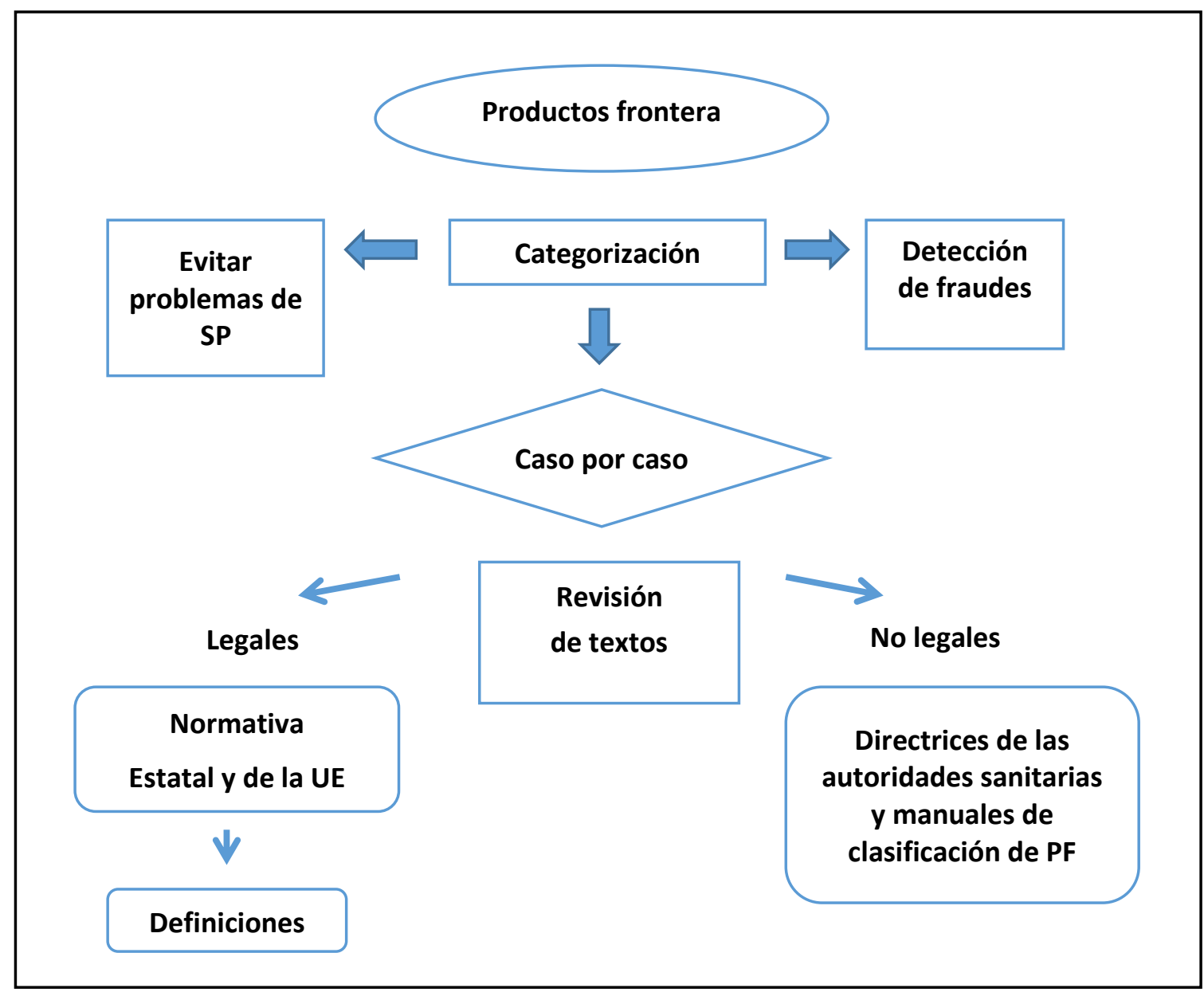

Figura 1 Estrategia de análisis para la clasificación de los productos frontera.

PF: Productos frontera; SP: Salud Pública; UE: Unión Europea;

\section{Resultados}

Los resultados obtenidos en la clasificación de los productos frontera se detallan en la Tabla I.

Tabla I Resultados de la clasificación caso por caso de los productos frontera

\begin{tabular}{|l|l|l|}
\hline \multicolumn{2}{|l|}{ PRODUCTOS SANITARIOS FRONTERA CON MEDICAMENTOS } \\
\hline $\mathbf{1}$ & Nombre & Apósito con Extracto de Capsicum frutescens \\
\cline { 2 - 3 } & Indicaciones & $\begin{array}{l}\text { Suministro de calor para el tratamiento de dolores neurológicos y } \\
\text { reumáticos. }\end{array}$ \\
\cline { 2 - 4 } & Resultado & $\begin{array}{l}\text { La capsaicina tiene un mecanismo de acción farmacológico. El } \\
\text { producto es un medicamento. }\end{array}$ \\
\hline $\mathbf{2}$ & Nombre & Crema/gel tópico con efecto analgésico \\
\cline { 2 - 4 } & Indicaciones & Tratamiento del dolor y las inflamaciones. \\
\cline { 2 - 4 } & Resultado & $\begin{array}{l}\text { El mecanismo de acción de los componentes mentol, alcanfor y } \\
\text { extractos de plantas medicinales es físico y farmacológico. Por el } \\
\text { principio de no acumulación y de jerarquía es un medicamento. }\end{array}$ \\
\hline $\mathbf{3}$ & Nombre & Crema con óxido de zinc \\
\cline { 2 - 4 } & Indicaciones & Prevención de las irritaciones menores de la piel. \\
\cline { 2 - 3 } & Resultado & $\begin{array}{l}\text { La acción farmacológica del óxido de zinc es accesoria a la acción } \\
\text { principal de barrera mecánica. Se trata de un producto sanitario. }\end{array}$ \\
\hline $\mathbf{4}$ & Nombre & Gotas oculares con lentes de contacto
\end{tabular}




\begin{tabular}{|c|c|c|}
\hline & \multirow{2}{*}{$\begin{array}{l}\text { Indicaciones } \\
\text { Resultado }\end{array}$} & \multirow{2}{*}{$\begin{array}{l}\text { Alivio de la irritación de los ojos por factores ambientales. } \\
\text { Se considera producto sanitario porque las gotas no estimulan la } \\
\text { secreción de las lágrimas ni curan ninguna patología. }\end{array}$} \\
\hline & & \\
\hline \multirow[t]{3}{*}{5} & Nombre & Producto con simeticona \\
\hline & Indicaciones & Para el meteorismo. \\
\hline & Resultado & $\begin{array}{l}\text { La simeticona disminuye la tensión superficial del gas. El } \\
\text { mecanismo de acción es físico. Se trata de un producto sanitario. }\end{array}$ \\
\hline \multicolumn{3}{|c|}{ PRODUCTOS SANITARIOS FRONTERA CON EQUIPOS DE PROTECCIÓN PERSONAL (EPI) } \\
\hline \multirow[t]{3}{*}{6} & Nombre & $\begin{array}{l}\text { Mascarilla con filtración de tipo II, referencia norma UNE EN } \\
14683\end{array}$ \\
\hline & Indicaciones & Para la protección frente al COVID-19. \\
\hline & Resultado & $\begin{array}{l}\text { Se trata de una Mascarilla quirúrgica y se diferencia de una } \\
\text { mascarilla EPI por su finalidad (protección de las personas } \\
\text { próximas frente a protección personal), mecanismo de acción } \\
\text { (filtra el aire exhalado frente a filtrar aire inhalado) y normas de } \\
\text { calidad (UNE EN } 14683 \text { frente a UNE EN 149). }\end{array}$ \\
\hline \multicolumn{3}{|c|}{ PRODUCTOS SANITARIOS FRONTERA CON FRONTERA CON COSMÉTICOS } \\
\hline \multirow[t]{3}{*}{7} & Nombre & Un blanqueador dental \\
\hline & Indicaciones & Para decoloraciones por patologías y tratamientos. \\
\hline & Resultado & $\begin{array}{l}\text { La decoloración no es una enfermedad, por tanto no se puede } \\
\text { considerar producto sanitario aunque hayan declarado una } \\
\text { finalidad médica. }\end{array}$ \\
\hline \multirow[t]{3}{*}{8} & Nombre & Agujas y tinta para tatuaje en medicina estética \\
\hline & Indicaciones & $\begin{array}{l}\text { Para la reconstrucción de la areola mamaria en casos de } \\
\text { mastectomía }\end{array}$ \\
\hline & Resultado & Se trata de un producto sanitario porque su finalidad es médica \\
\hline \multicolumn{3}{|c|}{ ACCESORIOS DE PRODUCTOS SANITARIOS FRONTERA CON COSMÉTICOS } \\
\hline \multirow[t]{3}{*}{9} & Nombre & Las pestañas y uñas postizas \\
\hline & Indicaciones & Para la decoración facial. \\
\hline & Resultado & $\begin{array}{l}\text { Las pestañas y uñas no son cosméticos pero van acompañados de } \\
\text { otros productos, mascara de pestañas y esmalte de uñas, que se } \\
\text { utilizan para cubrirlos y son considerados cosméticos decorativos. }\end{array}$ \\
\hline \multirow[t]{3}{*}{10} & Nombre & Maquinilla de afeitar con banda lubricante con Aloe vera \\
\hline & Indicaciones & Acción emoliente y protectora. \\
\hline & Resultado & $\begin{array}{l}\text { La maquinilla es un accesorio acompañado de un producto que } \\
\text { protege la piel y se considera en conjunto un producto cosmético. }\end{array}$ \\
\hline \multirow[t]{3}{*}{11} & Nombre & Sujetador impregnado de microcápsulas de Aloe vera \\
\hline & Indicaciones & Rejuvenece e hidrata la piel. \\
\hline & Resultado & $\begin{array}{l}\text { Se considera cosmético porque contiene sustancias que están en } \\
\text { contacto con la piel y la hidratan manteniéndola en buen estado. }\end{array}$ \\
\hline \multicolumn{3}{|c|}{ COSMÉTICOS FRONTERA CON ALIMENTOS } \\
\hline \multirow[t]{3}{*}{12} & Nombre & Comprimidos para chupar para la salud bucodental \\
\hline & Indicaciones & $\begin{array}{l}\text { Compuestos de cepas de Lactobacillus que mantienen la } \\
\text { microflora de la cavidad bucal y eliminan el mal aliento. }\end{array}$ \\
\hline & Resultado & $\begin{array}{l}\text { El producto responde a la definición de cosmético pero como su } \\
\text { finalidad es disolverse en la boca y tragarse por completo, se } \\
\text { considera alimento. }\end{array}$ \\
\hline \multicolumn{3}{|c|}{ COSMÉTICOS FRONTERA CON BIOCIDAS } \\
\hline \multirow[t]{2}{*}{13} & Nombre & $\begin{array}{l}\text { Gel hidroalcohólico desinfectante de manos con acción frente a } \\
\text { virus }\end{array}$ \\
\hline & Indicaciones & Limpiar las manos y protección frente a virus. \\
\hline
\end{tabular}




\begin{tabular}{|c|c|c|}
\hline \multicolumn{2}{|r|}{ Resultado } & Se trata de un biocida por sus alegaciones de carácter virucida. \\
\hline \multirow[t]{3}{*}{14} & Nombre & Crema protectora solar repelente de insectos \\
\hline & Indicaciones & $\begin{array}{l}\text { Barrera mecánica para prevenir enfermedades transmitidas por } \\
\text { insectos. }\end{array}$ \\
\hline & Resultado & $\begin{array}{l}\text { Su acción accesoria es de repelente de insectos, productos } \\
\text { considerados biocidas. Principio de jerarquía. }\end{array}$ \\
\hline \multicolumn{3}{|c|}{ COSMÉTICOS FRONTERA CON JUGUETES } \\
\hline \multirow[t]{3}{*}{15} & Nombre & Juguete con líquido jabonoso para hacer pompas de jabón \\
\hline & Indicaciones & Juego de niños. \\
\hline & Resultado & $\begin{array}{l}\text { Aunque el producto entra en contacto con la piel su finalidad no es } \\
\text { limpiar por tanto se considera un juguete. }\end{array}$ \\
\hline \multirow[t]{3}{*}{16} & Nombre & Muñeco infantil con productos de maquillaje \\
\hline & Indicaciones & Juego para maquillar al muñeco. \\
\hline & Resultado & $\begin{array}{l}\text { Se trata de un juguete cosmético porque cumple ambas } \\
\text { finalidades y debe ir etiquetado también como cosmético por las } \\
\text { sustancias químicas que contiene. }\end{array}$ \\
\hline \multirow[t]{3}{*}{17} & Nombre & Calcomanías o tatuajes lavables \\
\hline & Indicaciones & Dibujos decorativos para colocar sobre la piel. \\
\hline & Resultado & $\begin{array}{l}\text { Se tratan como cosméticos porque su finalidad es situarse sobre la } \\
\text { piel con el fin de modificar su aspecto aunque la tinta nunca llegue } \\
\text { a tocar la piel porque llevan una película de plástico que los } \\
\text { separa. }\end{array}$ \\
\hline
\end{tabular}

\section{Discusión de resultados}

En el presente trabajo se clasificaron los productos frontera dentro de una categoría de productos de acuerdo a diferentes criterios según el caso.

Cuando se trata de un caso dudoso entre dos categorías, el primer paso consiste en la consulta de ambas definiciones de productos en los textos legales. Al comparar vemos que hay algunos elementos que están presentes en ambas definiciones; en unos casos, no van a ser relevantes para la delimitación, pero en otros, van a ser precisamente los elementos claves para la misma [3].

\subsection{Productos sanitarios frontera con medicamentos}

La clasificación se realizó en base a elementos relevantes como el mecanismo de acción, si éste es farmacológico, inmunológico o metabólico se trata de un medicamento, tal como se clasificaron los apósitos con capsaicina, mientras que si estos mecanismos no existen como en los peeling químicos (acción química) o las gotas oculares con lentes de contacto (acción mecánica) o producto con simeticona para reducir el meteorismo (acción física) o solamente son accesorios al principal, se trata de un producto sanitario, es el caso de las cremas con óxido de zinc que tienen propiedades farmacológicas pero cuya acción principal es la de barrera mecánica para evitar irritaciones.

También se han tenido en cuenta elementos no relevantes como las funciones de restaurar, corregir o modificar las funciones fisiológicas comunes a los medicamentos como a los productos sanitarios. Las gotas oculares para las lentes de contacto no deben modificar significativamente las condiciones de funcionamiento del cuerpo humano. Si lo hacen, deberán considerarse como medicamentos [6]. 


\subsection{Productos sanitarios frontera con EPI}

Es esencial diferenciar entre productos que tengan la consideración de productos sanitarios (mascarillas quirúrgicas y batas quirúrgicas), equipos de protección individual EPI (ej. mascarillas FFP2), y mascarillas higiénicas. Para diferenciarlos se recurre a la finalidad, mecanismo de acción y normas de calidad. A cada tipo de producto le es aplicable un marco legislativo diferente. Para la puesta en el mercado de EPI debe cumplirse con las obligaciones del Reglamento (UE) 2016/425. Debe tenerse en cuenta que también existen equipos duales que cumplen la función de EPI y de Producto Sanitario (RD 1591/2009) y deben cumplir con las legislaciones aplicables a los dos ámbitos. La mascarilla higiénica es un tipo de producto que no entra en el ámbito de aplicación de Productos Sanitario ni de EPI. Por defecto no es necesario ningún tipo de autorización o certificación previa a la importación.

Para llevar a cabo una correcta clasificación se recurre a las definiciones de estos productos y a la información obligatoria que el Reglamento indica que deben de llevar estos productos: identificación del producto (marca y modelo), normas armonizadas UNE-EN-ISO, certificados de Conformidad Europea CE, instrucciones e información, etc. Esto nos permite detectar errores frecuentes de confusión entre estos tipos de productos de envasado y/o producto mal etiquetado o mal marcado con información incorrecta, o falta de ella.

\subsection{Productos sanitarios frontera con cosméticos}

El elemento relevante que permite distinguirlos es por su finalidad, si es sanitaria son productos sanitarios (Reglamento (UE) 2017/745) y en caso contrario son cosméticos.

Las agujas para tatuaje y las tintas con fines no sanitarios para maquillaje permanente, semipermanente o tatuaje (Productos de estética según la Disposición adicional Segunda del Real Decreto 209/2005), no son productos sanitarios.

En el caso de los blanqueadores dentales, el Manual de Productos Frontera y Clasificación, indica que si el producto blanqueante se utiliza en el interior de la cavidad dental o sobre la superficie de los dientes se trata de un producto cosmético. En España, la disposición adicional segunda del RD 209/2005 los clasifica como productos de cuidado personal.

\subsection{Accesorios de productos sanitarios frontera con cosméticos}

Las pestañas y uñas postizas junto con las máscaras y esmaltes forman parte de un todo, ya que estos productos solo pueden ser utilizados sobre este tipo de pestañas o uñas postizas, su finalidad es la decoración y son considerados como cosméticos.

En las maquinillas de afeitar con banda lubricante, la maquinilla se considera un soporte impregnado por un producto de acción lubricante para proteger la piel y mantenerla en buen estado por lo que debe de cumplir la normativa de productos cosméticos (Reglamento CE 1223/2009).

\subsection{Cosméticos frontera con alimentos}

Ambas legislaciones son excluyentes. En el artículo 2 del Reglamento 178/2002, la definición de alimento excluye a los cosméticos tal como los define la Directiva 76/768/CEE del Consejo. La orientación para distinguirlos está en el destino del producto. Serán alimentos si están destinados a ser ingeridos en su totalidad o si está destinado finalmente a ser ingerido tras ejercer su acción como en el caso de comprimidos para chupar para la salud bucodental. Y serán cosméticos si sólo se ingieren parcialmente por accidente como consecuencia de su aplicación en dientes y mucosa bucal o están destinados a ser absorbidos por la mucosa bucal. 


\subsection{Cosméticos frontera con biocidas}

La propagación del COVID-19 ha provocado un aumento del uso de limpiadores y desinfectantes para las manos en forma de geles, toallitas $\mathrm{u}$ otros productos que no se aclaran en agua. La correcta clasificación como cosmético o biocida va a depender de la presencia de una sustancia activa y de la finalidad principal del producto: La diferencia en la comercialización de los biocidas en España con respecto a los cosméticos estriba en que los primeros requieren de la concesión de una autorización previa por parte de la AEMPS como garantía de actividad desinfectante comprobada. El Reglamento (UE) n. 1223/2009 sobre los cosméticos regula los productos suministrados con una finalidad cosmética principal o exclusiva (limpieza de la piel, especialmente sin aclarado con agua). Los productos que contienen una sustancia activa y se suministran principalmente con un propósito biocida (es decir, destinados a controlar organismos nocivos) no están cubiertos por la legislación sobre cosméticos, por lo que entran dentro del ámbito de aplicación de la legislación en materia de biocidas (Reglamento (UE) n. ำ528/2012).

Los repelentes de insectos utilizados por los seres humanos no tienen acción médica, su mecanismo de acción principal es sobre los insectos y no sobre el cuerpo humano, con lo cual son biocidas que pertenecen al grupo principal 3 de plaguicidas; Tipo de producto 19: Repelentes y atrayentes y en España se regulan normativamente por el Real Decreto 1054/2002.

\subsection{Cosméticos frontera con juguetes}

Para poderlos distinguir y clasificar correctamente se compara la definición de cosmético con la de juguete que según la Directiva 2009/48 es todo producto diseñado o destinado, exclusivamente o no, para ser utilizado con fines de juego por niños menores de catorce años. Y además fijarse en la finalidad del producto, es posible que un producto tenga ambas finalidades Si es así, se aplican ambas directivas: no son excluyentes.

Si los productos se los aplican los niños, tienen la consideración de cosméticos además de juguetes.

Si los productos se aplican sobre los juguetes, busto de muñeco que se pinta, no son cosméticos, pero las sustancias y el etiquetado tiene que ser de un cosmético. (Reglamento CE 1223/2009).

\section{Conclusión}

La clasificación adecuada de un producto frontera que comparte características de dos categorías simultáneamente se realiza caso por caso recurriendo a las definiciones legales publicadas e incidiendo en aspectos clave diferenciadores tales como mecanismo de acción, finalidad, modo de administración y lugar de aplicación. También se tienen en cuenta:

- La consideración de elementos legales adicionales (jerarquías) y las exclusiones legales.

- Las características del producto: indicaciones, etiquetado, normas de calidad y si existen varios componentes, se diferencia entre la acción principal y la acción coadyuvante y/o auxiliar.

Con este procedimiento se consigue la detección de errores de clasificación o fraudes por la intención de obviar los trámites de autorizaciones más estrictas de los medicamentos respecto a alimentos, productos sanitarios, cosméticos y productos de cuidado personal cuya comercialización es más económica y sencilla constituyendo un asunto prioritario de salud pública. La clasificación de un medicamento como otro tipo de producto supone una indefensión de los enfermos al que van destinados, ya que los medicamentos no son inocuos y pueden producir efectos perjudiciales de los que es necesario advertir. 


\section{ANEXO I Legislación consultada}

\subsection{Legislación española}

Ley 28/2009, de 30 de diciembre, de modificación de la Ley 29/2006, de 26 de julio, de garantías y uso racional de los medicamentos y productos sanitarios. (BOE núm. 315, de 31 de diciembre).

Ley 10/2013, de 24 de julio, por la que se incorporan al ordenamiento jurídico español las Directivas 2010/84/UE del Parlamento Europeo y del Consejo, de 15 de diciembre de 2010, sobre farmacovigilancia, y 2011/62/UE del Parlamento Europeo y del Consejo, de 8 de junio de 2011, sobre prevención de la entrada de medicamentos falsificados en la cadena de suministro legal, y se modifica la Ley 29/2006, de 26 de julio, de garantías y uso racional de los medicamentos y productos sanitarios. (BOE 177, de 25 de julio).

Real Decreto 1662/2000, de 29 de septiembre, sobre productos sanitarios para diagnóstico "in vitro". (BOE núm. 235, de 30 de septiembre).

Real Decreto 1054/2002, de 11 de octubre, por el que se regula el proceso de evaluación para el registro, autorización y comercialización de biocidas. (BOE núm. 247, de 15 de octubre).

Real Decreto 209/2005, de 25 de febrero, por el que se modifica el RD 1599/1997, sobre productos cosméticos.

Real Decreto 1143/2007, de 31 de agosto, por el que se modifican los Reales Decretos 634/1993, de 3 de mayo, sobre productos sanitarios implantables activos; 414/1996, de 1 de marzo, por el que se regulan los productos sanitarios; y 1662/2000, de 29 de septiembre, sobre productos sanitarios para diagnóstico in vitro (BOE núm. 210, de 1 de septiembre). Artículo tercero.

Real Decreto 1591/2009, de 16 de octubre, por el que se regulan los productos sanitarios (BOE núm.268, de 6 de noviembre).

Real Decreto 1616/2009, de 26 de octubre, por el que se regulan los productos sanitarios implantables activos (BOE núm.268, de 6 de noviembre).

Real Decreto 1090/2010, de 3 de septiembre, por el que se modifica el Real Decreto 1054/2002, de 11 de octubre, por el que se regula el proceso de evaluación para el registro, autorización y comercialización de biocidas. (BOE núm. 224, de 15 de septiembre).

Real Decreto 1275/2011, de 16 de septiembre, por el que se crea la Agencia estatal "Agencia Española de Medicamentos y Productos Sanitarios" y se aprueba su Estatuto (BOE núm. 229, de 23 de septiembre).

Real Decreto 1193/2012, de 3 de agosto, por el que se modifica el Real Decreto 1662/2000, de 29 de septiembre, sobre productos sanitarios para diagnóstico «in vitro». (BOE núm. 186, de 4 de agosto).

Real Decreto Legislativo 1/2015, de 24 de julio, por el que se aprueba el texto refundido de la Ley de garantías y uso racional de los medicamentos y productos sanitarios. (BOE núm. 177, de 25 de julio de 2015).

Corrección de errores del Real Decreto Legislativo 1/2015, de 24 de julio, por el que se aprueba el texto refundido de la Ley de garantías y uso racional de los medicamentos y productos sanitarios. (BOE núm. 306, de 23 de diciembre de 2015).

Real Decreto 85/2018, de 23 de febrero, por el que se regulan los productos cosméticos. (BOE núm. 51, de 27 de febrero).

Resolución, de 19 de febrero de 2013, de la Agencia Española de Medicamentos y Productos Sanitarios, por la que se crea la sede electrónica de la Agencia Estatal "Agencia Española de Medicamentos y Productos Sanitarios". (BOE núm. 57, de 7 de marzo).

Orden SPI/1864/2011, de 20 de junio, por la que se autoriza la presentación a través del registro electrónico del departamento de determinados escritos, comunicaciones y solicitudes en materia de productos sanitarios, cosméticos y productos de higiene personal de la Agencia Española de Medicamentos y Productos Sanitarios. 


\subsection{Legislación europea}

Reglamento (CE) no 178/2002 del Parlamento Europeo y del Consejo de 28 de enero de 2002, por el que se establecen los principios y los requisitos generales de la legislación alimentaria, se crea la Autoridad Europea de Seguridad Alimentaria y se fijan procedimientos relativos a la seguridad alimentaria (DO L 31 de 1.2.2002, p. 1)

Reglamento(UE) no 1235/2010 del Parlamento Europeo y del Consejo de 15 de diciembre de 2010, que modifica, en lo que respecta a la farmacovigilancia de los medicamentos de uso humano, el Reglamento (CE) no 726/2004, por el que se establecen procedimientos comunitarios para la autorización y el control de los medicamentos de uso humano y veterinario y por el que se crea la Agencia Europea de Medicamentos, y el Reglamento (CE) no 1394/2007 sobre medicamentos de terapia avanzada (Diario Oficial de la Unión Europea L 348:1-16, de 31/12/2010).

Reglamento (UE) no 528/2012 del Parlamento Europeo y del Consejo de 22 de mayo de 2012, relativo al uso y comercialización del uso de biocidas. (DO 167 de 27.06.2012, p. 123).

Reglamento (UE) no 334/2014 del Parlamento Europeo y del Consejo de 11 de marzo de 2014, por el que se modifica el Reglamento (UE) no 528/2012, relativo a la comercialización y el uso de los biocidas, en relación con determinadas condiciones de acceso al mercado. (DO 103 de 5.4.2014, p2).

Reglamento (UE) no 2283/2015 del Parlamento Europeo y del Consejo de 25 de noviembre de 2015, relativo a los nuevos alimentos, por el que se modifica el Reglamento (UE) no 1169/2011 del Parlamento Europeo y del Consejo y se derogan el Reglamento (CE) no 258/97 del Parlamento Europeo y del Consejo y el Reglamento (CE) no 1852/2001 de la Comisión (DO L327/1 ES de 11.12.2015).

Reglamento (UE) no 425/2016 del Parlamento Europeo y del Consejo de 9 de marzo de 2016, relativo a los equipos de protección individual y por el que se deroga la Directiva 89/686/CEE del Consejo. (DO L81/51 ES de 31.3.2016).

Reglamento (UE) 2017/745 del Parlamento Europeo y del Consejo, de 5 de abril de 2017, sobre los productos sanitarios, por el que se modifican la Directiva 2001/83/CE, el Reglamento (CE) n. ${ }^{\circ}$ 178/2002 y el Reglamento (CE) n. ${ }^{\circ} 1223 / 2009$ y por el que se derogan las Directivas 90/385/CEE y 93/42/CEE del Consejo (DO 117, de 5.5.2017, p. 1).

Reglamento (UE) 2017/746 del Parlamento Europeo y del Consejo, de 5 de abril de 2017, sobre los productos sanitarios para diagnóstico in vitro y por el que se derogan la Directiva 98/79/CE y la Decisión 2010/227/UE de la Comisión (DO 117, de 5.5.2017, p. 176).

Reglamento (CE) No 1394/2007 del Parlamento Europeo y del Consejo de 13 de noviembre de 2007, sobre medicamentos de terapia avanzada y por el que se modifican la Directiva 2001/83/CE y el Reglamento (CE) 726/2004. (DO L 324 de 10.12.2007, p. 121).

Reglamento (CE) № 1223/2009 del Parlamento Europeo y del Consejo de 30 de noviembre de 2009, sobre los productos cosméticos (DO 342 de 22.12.2009, p.59).

Directiva 65/65/CEE del Consejo, de 26 de enero de 1965, relativa a la aproximación de las disposiciones legales, reglamentarias y administrativas, sobre especialidades farmacéuticas (DO 22 de 9.2 .1965 , p. 369/373).

Directiva 93/42/CEE del Parlamento Europeo y del Consejo de 14 de junio de 1993, relativa a los productos sanitarios. (DO L 169 de 12.7.1993, p. 1).

Directiva 2001/83/CE del Parlamento Europeo y del Consejo, de 6 de noviembre de 2001, por la que se establece un código comunitario sobre medicamentos para uso humano (DO L 311 de 28.11.2001, p. 67).

Directiva 2004/23/CE del Parlamento Europeo y del 31 de marzo de 2004, relativa al establecimiento de normas de calidad y de seguridad para la donación, la obtención, la evaluación, el procesamiento, la preservación, el almacenamiento y la distribución de células y tejidos humanos (DO L 102 de 7.4.2004, p. 48).

Directiva 2004/27/CE del Parlamento Europeo y del Consejo de 31 de marzo de 2004, que modifica la Directiva 2001/83/CE por la que se establece un código comunitario sobre medicamentos de uso humano (Diario Oficial de la Unión Europea L 136/34 ES de 30.4.2004). 
Directiva 2009/48/CE del Parlamento Europeo y del Consejo de 18 de junio de 2009 sobre la seguridad de los juguetes. (DO L 170 de 30.6.2009, p. 1/37).2. Material y Métodos

Contribución de los autores: Bernardo Prieto Muñoz: contribución importante a la idea y título del trabajo, introducción, referenciación bibliográfica y aprobar versión final. María del Carmen Vidal Miñana: análisis, interpretación y discusión de resultados, participación en la redacción del borrador del artículo.

\section{Referencias Bibliográficas}

1. AEMPS (2018). Carta de servicios 2018-2021. (Internet). Disponible en: https://www.aemps.gob.es/laAEMPS/cartaDeServicios/docs/Carta-de-servicios-AEMPS.pdf

2. Amarilla Mateu N. y Lozano Arjona M. (2016). Productos Frontera, Productos Milagro y la protección de la salud pública. Derecho y salud, ISSN 1133-7400, Vol. 26, №. Extra 1, 2016 (Ejemplar dedicado a: XXV Congreso 2016: El avance de las Ciencias de la Salud y las incertidumbres del Derecho), págs. 237-246. Disponible en: https://www.ajs.es/descarga/attach/898

3. Abad Luna MC. (2013). La Frontera en productos sanitarios. Industria farmacéutica. 2013 Ene-Feb; (175):3035. Disponible en: https:// www.aemps.gob.es/publicaciones/articulo/dcs/Ind-Fcia-Frontera-ps.pdf

4. EMA (2019) Manual on borderline and classification in the community regulatory framework for medical devices, Version 1.22 (05-2019). Disponible en: https://ec.europa.eu/docsroom/documents/35582

5. EMA (2020) Borderline Manual febrary 2020, Version 5 (02 2020). Disponible en: https://ec.europa.eu/docsroom/documents/40041

6. Faus y Molines (1998). Algunas consideraciones sobre los productos frontera. Cápsulas' no 13; marzo 1998. Disponible en:

(C) 2021 por los autores; Esta obra está sujeta a la licencia de Reconocimiento 4.0

Internacional de Creative Commons. Para ver una copia de esta licencia, visite http://creativecommons.org/licenses/by-nc-nd/4.0/. 